\title{
Motorcycle Helmet Noise and Active Noise Reduction
}

\author{
Charles H. Brown ${ }^{*}, 1$ and Michael S. Gordon ${ }^{2}$ \\ ${ }^{1}$ University of South Alabama, 307 University Blvd. Mobile, AL 36688-0002, USA \\ ${ }^{2}$ William Patterson University, 300 Pompton Rd. Wayne, NJ 07470, USA
}

\begin{abstract}
The incidence of collisions between motorcyclists and other vehicles may be significantly reduced by research that improves the acoustic awareness of cyclists, and thus heightens the ability of cyclists to respond to unexpected incursions from the surrounding traffic. We use our hearing as an early warning system, and hearing swiftly redirects our vision and attention. This shift in gaze is critical to our capacity to assess the location, direction of travel, and velocity of approaching vehicles. The present study was composed of two experiments. In the first experiment a Neumann KU-100 dummy head with embedded binaural microphones was used to measure noise levels in a motorcycle helmet as a function of velocity. Noise levels were measured in two helmets, one with active noise reduction technology, and one without. The results showed that noise levels exceeded $100 \mathrm{~dB}(\mathrm{~A})$ at highway speeds in the absence of noise reduction technology. The helmet with active noise control ear muffs was able to attenuate helmet noise by up to $26 \mathrm{~dB}$. Active noise control technology shows great promise for noise reduction for the motorcycle helmet industry, and the development of "quiet" helmets is important for both hearing conservation and highway safety. The second experiment surveyed subjective perceptions of helmet noise by motorcyclists. The results from the present sample showed that $92.1 \%$ of the respondents objected to the high noise levels associated with cycling, $63.5 \%$ wore earplugs, $46.8 \%$ reported tinnitus, and $95.2 \%$ wanted a quieter helmet.
\end{abstract}

Keywords: Motorcycle noise, helmet noise, active noise control, motorcycle safety, highway safety, hearing conservation.

\section{INTRODUCTION}

The hearing abilities of all cyclists are severely compromised by the twin impediments of engine noise and wind turbulence noise. Motorcycle helmet noise is of concern for both long-term hearing conservation and for its impact on the acoustic awareness of cyclists, and hence on highway safety. Several studies suggest that occupational motorcyclists such as police officers are at risk of noise induced hearing loss [1-7]. Furthermore, high and even moderate noise levels impair reaction times, impede attention, and reduce the effectiveness of perceptual and behavioral responses pertinent to highway safety [8-11]. In the US the incidence of motorcycle fatalities has increased 4fold over the past 15 years [12]. In part this risk factor seems to have increased due to an increase in motorcycle size, and an increase in the number of novice cyclists $[13,14]$; and this trend is apt to accelerate with the expectation of progressive increments in the cost of gasoline.

The majority of motorcycle accidents are not caused by speeding or irresponsible driving on the part of the motorcyclist: most accidents are caused by passenger vehicles violating the right-of-way of cyclists $[12,15]$. However, the incidence of collisions between motorcyclist and other vehicles may be significantly reduced by research that improves the acoustic awareness of cyclists, and thus

*Address correspondence to this author at the University of South Alabama, 307 University Blvd., Mobile, AL 36688-0002, USA; Tel: 251-460-6372; Fax: 251-460-6320; E-mail: cbrown@usouthal.edu heightens the ability of cyclists to hear highway warning sounds, and respond to unexpected incursions from the surrounding traffic. We use our hearing as an early warning system, and hearing swiftly redirects our vision and this shift in gaze is critical to our capacity to assess the location, direction of travel, and velocity of approaching vehicles. Furthermore, visual perception has been found to be more accurate as well as faster when the visual stimulus is paired with an auditory cue [16-18].

It is likely that the design of some motorcycle helmets hampers the hearing of cyclists under some conditions [1923], and an unknown number of cyclists use ear plugs to attenuate the disturbing noise levels associated with the design of many current motorcycle helmets. Earplugs reduce helmet noise, and they provide some protection for noise induced hearing loss [24, 25], but they impair the ability of cyclists to hear sirens and other highway warning sounds under some conditions [26]. In the US earplugs are legal in some jurisdictions, and illegal in others. At present cyclists have only a limited ability to use their auditory system to help them negotiate traffic and avoid collisions, and though it is recognized that the noise associated with cycling may have an adverse impact on safety [12, 13, 27], there are no established protocols for measuring hearing in motorcycle helmets in the US, and there is no systematic research program designed to encourage the helmet industry to apply active noise-reduction and signal-processing technologies to address this problem area for hearing conservation and highway safety. 
An acoustic testing protocol for motorcycle helmets focused principally on hearing conservation has been proposed in Europe [28]. There is reason to believe that a comprehensive acoustic testing protocol can be developed that promotes both hearing conservation and highway safety objectives for the US. The present research seeks to develop a methodology intended to systematically measure hearing in motorcycle helmets, provide baseline measurements that can be used to evaluate the severity of the noise levels cyclists' currently encounter, test the utility of electronic ear muffs of modern design for helmet noise abatement, and survey the subjective perceptions of motorcyclists towards helmet noise, and the use of earplugs.

In the present study we used a half-helmet style motorcycle helmet. This is the most popular helmet style in the US. V-twin cruiser style motorcycles are the most popular motorcycles in use in the US today, and it is relatively uncommon to see other helmet styles used by the cruiser cycling community. The half-helmet style permits good ventilation and unobstructed hearing (without removing the helmet) when riders are at an intersection, or at low velocities, but they provide limited shielding of the ears at moderate and high velocities. The relationship between helmet style and helmet noise is complicated because the aerodynamic parameters of the motorcycle's design are major contributors to the noise levels measured within the helmet [6, 29-32]. Lower and his associates [31, 32] studied helmet noise as a function of changes in the height of the motorcycle wind screen. They found that the relative ranking of helmet noise level, from the quietest helmet to the noisiest helmet, changed when the windscreen height was altered. The quietest helmet measured with a low windscreen setting became the nosiest helmet when the windscreen was raised. Conversely, one helmet in the set that was the nosiest at the low windscreen height became one of the quietest at the elevated windscreen position. Thus, it was not possible to meaningfully rank helmets as noisy or quiet, because variations in the air stream around the cyclist's head associated with different motorcycle designs and windscreen configurations had a strong impact on the noise levels recorded in the same helmet. Jordon and his associates [6] also measured noise levels in different style of motorcycle helmets. They observed lower noise levels in some openface helmets relative to some full-face helmets, but it is unclear if these noise level differences would be systematically observed on different motorcycles with different aerodynamic properties. Lower and his colleagues $[31,32]$ noted that many cyclists prefer a windscreen height that keeps the wind off the chest, and presents an unobstructed view of the road. This configuration places the chin of the rider near the top of the windscreen, and Lower and his associates found that this positioned the helmet in a stream of very turbulent air that tended to elevate helmet noise levels.

At highway speeds $(100 \mathrm{~km} / \mathrm{h}$ or more) noise levels in excess of $100 \mathrm{~dB}$ (A) will be encountered in virtually all styles of helmets on all types of motorcycles. At these speeds it can be assumed that wind turbulence is the most prominent source of noise, although there is still a contribution of engine vibration, road noise, and related sources. Noise at this level may approach the threshold of discomfort for some listeners, and many riders will experience tinnitus and/or a temporary hearing loss. Sustained exposure to noise at this level is linked to permanent hearing loss [33]. It is likely that many cyclists in the US use ear plugs when commuting at highway speeds irrespective of the legality of earplug usage in their jurisdiction.

\section{EXPERIMENT 1: ACOUSTIC MEASUREMENT OF HELMET NOISE AS A FUNCTION OF VELOCITY}

This study was designed to measure the noise level and noise spectrum cyclists experience as a function of velocity in helmets with and without active noise suppression. Clearly at $0 \mathrm{~km} / \mathrm{h}$ the only noise source is the exhaust note of the idling motorcycle. The intensity and spectrum of engine vibration at idle varies dramatically across cycles, but tends to have the greatest energy below $500 \mathrm{~Hz}$ and from 40 to 95 $\mathrm{dB}-\mathrm{A}$ depending on the size and make of the engine. As the cycle begins to accelerate the noise will increase due to wind turbulence in addition to the exhaust note produced by the motor rotating at higher rpm levels. At the highest velocities wind turbulence is very high, and for most motorcycles engine noise is likely a negligible contributor to helmet noise levels relative to the magnitude of wind noise.

\section{Materials and Methods}

A dummy head engineered to simulate an adult male human head (Neumann KU-100) was positioned on a motorcycle tank bag located on the gas tank of a motorcycle. The position of the dummy head approximates the location of the head for a motorcyclist on a sport bike riding in the "tucked" position. The Neumann KU-100 is a binaural microphone system with microphones located in the ear canal near the position of the ear drum (tympanic membrane). The Neumann KU-100 instrumentation was designed to conduct acoustic recordings and sound measurements that simulate human hearing. Acoustic measurements were conducted with the dummy head fitted with a motorcycle helmet (see Fig. 1), and with a half-helmet fitted with an electronic noise cancelling ear muff (Fig. 2). A half-helmet was selected for these measurements because this helmet style is the most widely used. The Noisebuster PA4200: Hard Hat Cap Mount (Pro Tech Technologies, Inc.) active noise control (ANC) electronic noise-cancelling ear muff employed was used. This muff is ANSI certified [25 $\mathrm{dB}$ NPR/CSA Class A/SLC (80) 24.7 Class 4], and achieves a 25 $\mathrm{dB}$ noise reduction rating. Many active noise control ear muffs in the marketplace are designed to permit unobstructed hearing for moderate and low-amplitude sounds, but attenuate high-amplitude sounds. The Noisebuster PA4200 is intended for use in industrial settings where the user may experience significant fluctuations in noise levels as machinery is turned on or off.

We used two helmets, a Fulmer Ranger (Fig. 1), and a Fulmer AF-80 (Fig. 2). These models are used locally by the Motorcycle Safety Foundation for rider safety classes, and they were selected because they fit the dimensions of the Neumann dummy head and the active noise control ear muff. 
An audio signal from the left channel (left ear) of the Neumann KU-100 dummy head was recorded with a Tascam DR-100 portable digital field recorder positioned in the tank bag. Sound samples were recorded as 16 bit wav files at a sample rate of $44.1 \mathrm{kHz}$. The left channel of the KU-100 dummy head was calibrated according to the Neumann KU100 specifications. The left ear of the dummy head was removed exposing the microphone capsule, a Brüel and Kjær type 4230 sound level calibrator was positioned to encase the microphone diaphragm and the calibrator supplied a $1 \mathrm{kHz}$ calibration tone at $93.6 \mathrm{~dB}$, the reference level used for free field measurements. Thus, the sound levels reported here are in reference to this calibration tone. The right channel of the Tascam recorder was connected to a Sony WCS-999 wireless microphone system permitting the motorcycle operator to announce the beginning and ending of the sound samples and the velocity at which each sample was conducted. Recordings were conducted on a Kawasaki EX500 (Ninja 500R) motorcycle. A Garmin Map 60 global positioning system was used to calibrate the Kawasaki speedometer. The digital sound files were transferred to a Dell Optiplex GX620 desk top computer, edited by Cool Edit Pro 2.0 sound editing software, and sound frequency and amplitude measurements were conducted with True RTA software using a calibrated Behringer UF0202 audio interface. Daqarta signal processing software was used to convert unweighted sound level measurements [dB (flat)] to the $\mathrm{dB}(\mathrm{A})$ scale.

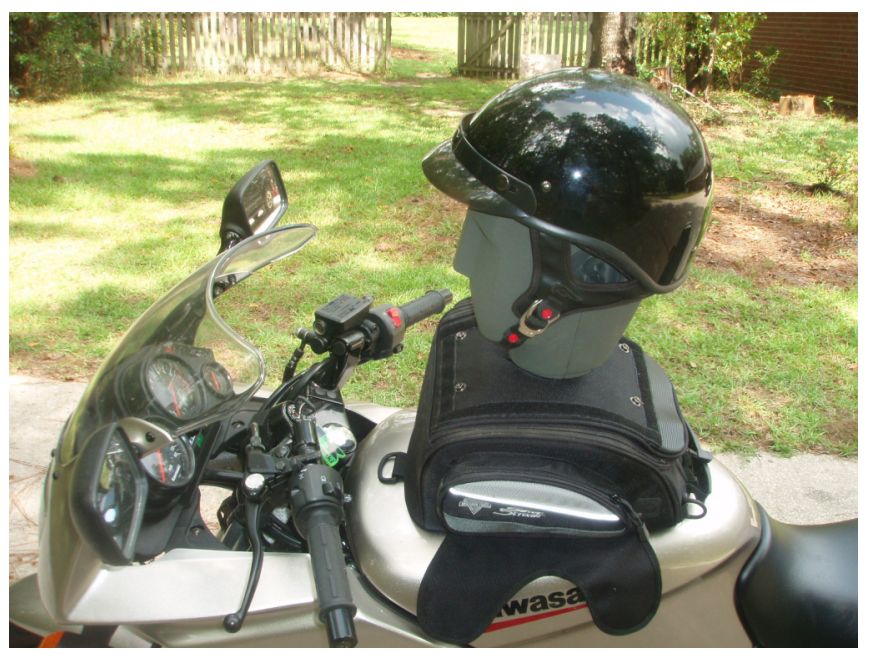

Fig. (1). Neumann KU-100 acoustically engineered dummy head with a binaural microphone system positioned at the location of the tympanic membrane. The dummy head is shown with a half-helmet, the most popular style of helmet.

\section{Procedure}

Audio recordings were conducted at seven velocities: 0 , $20,40,60,80,100,120 \mathrm{~km} / \mathrm{h}$. At $0 \mathrm{~km} / \mathrm{h}$ the motorcycle was at idle (1100 rpm). The Kawasaki speedometer was checked for accuracy with the Garmin global positioning system at 20 $\mathrm{km} / \mathrm{h}$ intervals from $20 \mathrm{~km} / \mathrm{h}$ to $120 \mathrm{~km} / \mathrm{h}$. Speedometer error was very small (less than $4 \%$ ) in the range tested, and the targeted velocities were indexed by lines drawn on transparency film taped to the face of the speedometer. A complete data set was recorded at two different calibrated gain settings on the Tascam recorder. For each gain setting two sets of recordings were recorded for each velocity. All measurements were conducted in a rural setting. When the motorcycle achieved a target velocity the operator announced the velocity and spoke "begin sample", maintained the velocity for the sample interval, and then spoke "end sample". Recorded sound files were edited to produce a 10-20 second long sound sample. These samples were subsequently played as a continuous loop into the TrueRTA sound analysis system. For our measurements TrueRTA was set to average 100 successive samples, and this yielded stable repeatable values. The Neumann KU-100 is able to record signals up to $135 \mathrm{~dB}$, a value greater than that encountered in the present study; the frequency response of the KU-100 is flat $+/-2 \mathrm{~dB}$ from $50 \mathrm{~Hz}$ to $10 \mathrm{kHz}$, the range selected for the present study.

TrueRTA provides flat or unweighted sound pressure level measurements which are optimal for evaluating noise levels across the audio spectrum, but this software does not have a provision for converting sound level measurements to the $\mathrm{dB}$ (A) scale. We imported the audio files into Daqarta, a signal processing program, to convert our unweighted sound level measurements $\mathrm{db}$ (flat) to the $\mathrm{dB}(\mathrm{A})$ scale.

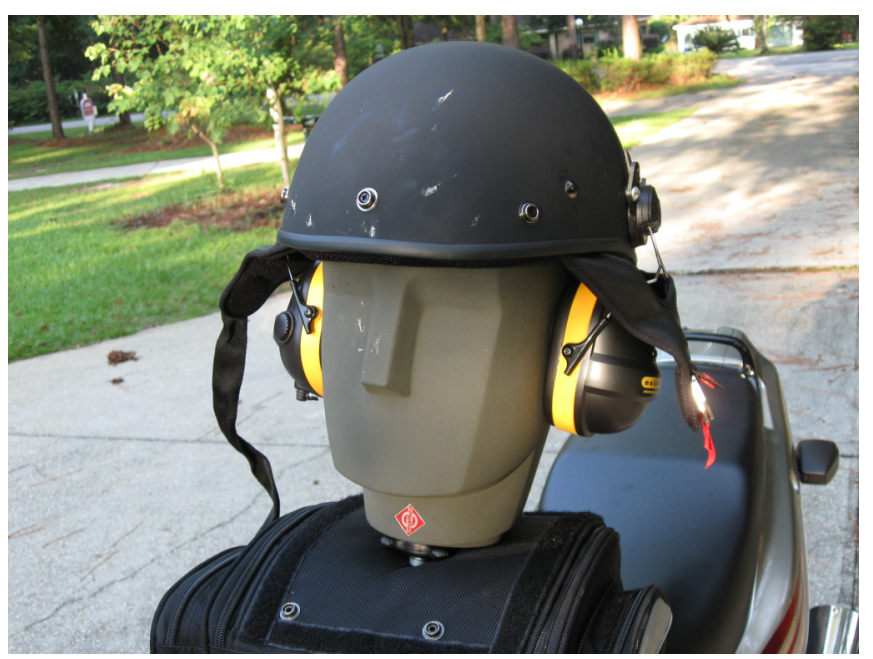

Fig. (2). Half-helmet with an ANSI certified [25 dB NPR/CSA Class A/SLC (80) 24.7 Class 4] active noise control (electronic noise cancelling) earmuff.

\section{RESULTS AND DISCUSSION}

\section{Half-Helmet without Hearing Protection}

In the absence of hearing protection the noise associated with cycling was substantial. At idle a sound level of $77 \mathrm{~dB}$ was observed in the $1 / 3^{\text {rd }}$ octave band centered at $100 \mathrm{~Hz}$. The noise associated with motorcycling increased with velocity, and became prominent for velocities above 40 $\mathrm{km} / \mathrm{h}$. At a velocity of $120 \mathrm{~km} / \mathrm{h}$ (about $75 \mathrm{mph}$ ) noise levels of $108 \mathrm{~dB}$ were obtained in $1 / 3^{\text {rd }}$ octave bands centered at $100 \mathrm{~Hz}, 125 \mathrm{~Hz}$, and $200 \mathrm{~Hz}$ (Fig. 3). Furthermore, at 120 $\mathrm{km} / \mathrm{h}$ the observed noise levels exceeded $100 \mathrm{~dB}$ for all ten $1 / 3^{\text {rd }}$ octave bands sampled from $50 \mathrm{~Hz}$ to $400 \mathrm{~Hz}$. TrueRTA calculated a value of $118.1 \mathrm{~dB}$ for the total spectrum of the noise sample using a linear $\mathrm{dB}$ (flat) scale. Fig. (3) also shows that the noise level peaks occurred in the low- 
frequency range (200 $\mathrm{Hz}$ or less). Thus, as velocity increased, the amplitude of the noise increased, and the peak frequency of the noise also tended to increase. At $0 \mathrm{~km} / \mathrm{h}$ engine noise is the sole source of noise as the motorcycle is stationary. At $20 \mathrm{~km} / \mathrm{h}$ the noise spectrum is very similar to that recorded at $0 \mathrm{~km} / \mathrm{h}$, and engine noise is likely the prime source of the noise at this velocity as well. At $40 \mathrm{~km} / \mathrm{h}$ and above, the shape of the noise spectrum changed and the spectrum exhibited a uniform pattern for changes in velocity. This observation suggests that wind turbulence is the prime source of the noise recorded for velocities of $40 \mathrm{~km} / \mathrm{h}$ and above. We measured engine noise at 1700, 3000, 3900, 5000 , and $6200 \mathrm{rpm}$ which corresponded to the all the target velocities used in the present study. The result showed that at $40 \mathrm{~km} / \mathrm{h}$ and above the engine noise was at least $18 \mathrm{~dB}$ below the recorded noise level and hence the observed noise was largely due to wind turbulence on this motorcycle. The engine noise produced by some motorcycles is much louder than that generated by the Kawasaki EX500, and engine noise produced by these motorcycles would likely contribute to the helmet noise levels recorded at higher velocities compared to those observed here.

As shown in Fig. (3) noise levels tended to decrease at a rate of about $10 \mathrm{~dB}$ per octave in the range of $250 \mathrm{~Hz}$ to 8 $\mathrm{kHz}$. At $60 \mathrm{~km} / \mathrm{h}$ and above, the peak sound pressure level increased only $7 \mathrm{~dB}$ (from $101 \mathrm{~dB}$ to $108 \mathrm{~dB}$ ), but the perceived noise levels cyclist experience is likely to be much greater. This is due to the fact that uniformly from $200 \mathrm{~Hz}$ to $10,000 \mathrm{~Hz}$, the noise level increased about $20 \mathrm{~dB}$ as velocity was incremented from $60 \mathrm{~km} / \mathrm{h}$ to $120 \mathrm{~km} / \mathrm{h}$. Fig. (4) displays a waveform created by splicing together 2 second noise samples recorded at each of the seven target velocities from $0 \mathrm{~km} / \mathrm{h}$ to $120 \mathrm{~km} / \mathrm{h}$. In agreement with the peaks of the spectral data shown in Fig. (3), only small differences in the amplitude of the waveform are observed for the samples recorded at $80 \mathrm{~km} / \mathrm{h}, 100 \mathrm{~km} / \mathrm{h}$, and $120 \mathrm{~km} / \mathrm{h}$ (the last 6 seconds displayed in Fig. 4). The reader can hear these noise samples by clicking on the speaker icon and perceptually observe how the perceived loudness increases significantly with increments in velocity even though the measured increments in amplitude show only a modest change. What this means is that on paper the increment in noise amplitude between $100 \mathrm{~km} / \mathrm{h}$ and $120 \mathrm{~km} / \mathrm{h}$, for example, is small, but to the motorcyclist the perceived increment in helmet noise is huge.

Many motorcyclists prefer a windscreen that deflects the wind from the chest, yet permits an unobstructed view of the road. In this configuration the chin of the rider is located near the top of the windscreen, and Lower and his associates observed that this arrangement positioned the helmet in highly turbulent air and tended to elevate noise levels [31, 32]. In the present study the Neumann KU-100 dummy head was positioned in a similar location (relative to the fairing on a sport bike), and we likely encountered turbulence and helmet noise levels similar to those noted by Lower. The present data measured with the Neumann KU-100 dummy head is very similar to other studies which have placed a microphone inside a helmet next to the ear of a motorcyclist (Fig. 5). Helmet noise increases with velocity and typically exceeds levels of $100 \mathrm{~dB}(\mathrm{~A})$ at velocities above $100 \mathrm{~km} / \mathrm{h}$. These results suggest that the Neumann KU-100 is a good instrument for measuring noise abatement strategies in

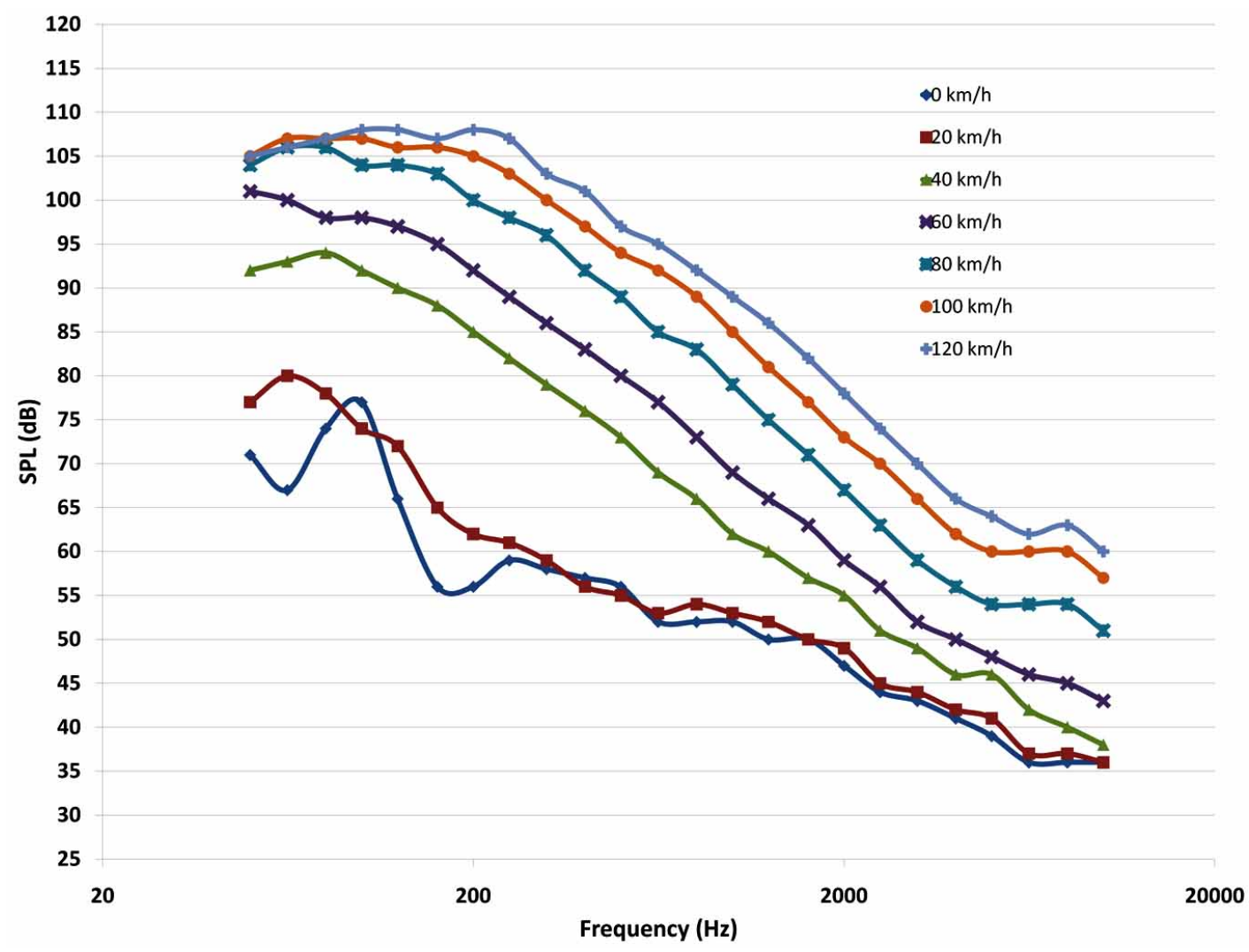

Fig. (3). Sound pressure levels as a function of velocity for a half helmet without hearing protection. 


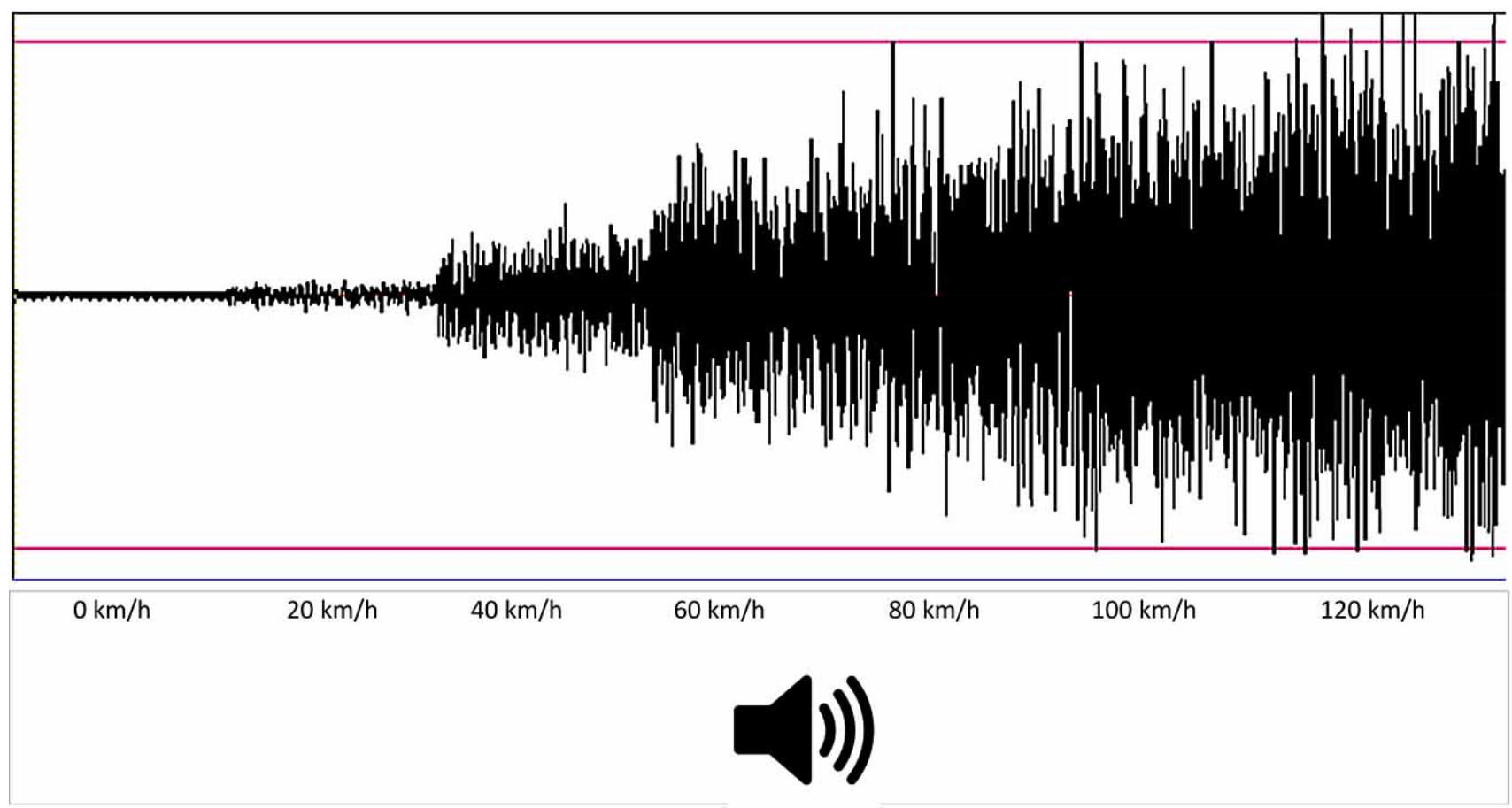

Fig. (4). Waveform created by splicing two-second noise sample for all seven velocities from $0 \mathrm{~km} / \mathrm{h}$ to $120 \mathrm{~km} / \mathrm{h}$ for samples recorded with the half helmet without hearing protection.

motorcycle helmets, and that the recordings obtained are ecologically valid approximations of the acoustic experience motorcyclists encounter while traveling at various velocities, on different motorcycles with different helmets. It is possible that differences in the head and neck anatomy of different motorcyclist and the interplay between variations in human anatomy and the helmet is one source of variation that can complicate the assessment of various noise abatement strategies. The development of a uniform platform and protocol for helmet testing would allow different laboratories to replicate and compare data sets, and this would stimulate product development and testing in the active noise control and safety helmet industries.

The present results show that the noise spectrum is greatest at $200 \mathrm{~Hz}$ and below. Though the frequency peak for the helmet noise may vary depending on the aerodynamic properties of the helmet and motorcycle being studied, researchers have consistently observed that the principle problem for helmet noise control is low-frequency noise abatement $[25,35,36]$. The optimal design for an acoustically engineered motorcycle helmet would attenuate wind turbulence noise, but permit good hearing for other portions of the audio spectrum. This would shield the rider from excess noise exposure, and yet permit the rider to clearly hear highway safety sounds. Helmet noise is not like white noise (or pink noise) which affects the entire audio spectrum. Helmet noise is largely confined to low frequencies, and because wind turbulence generates a steady noise at steady velocities, it may be possible to design an acoustically smart helmet that shields the rider from excess low-frequency noise exposure to continuous noises, but permits good hearing for intermittent signals (such as horns and sirens), or for signals that are above the frequency range associated with wind noise.

\section{Half Helmet Condition with Active Noise Control Hearing Protection}

The active noise control (ANC) ear muff strongly changed the noise levels associated with cycling. Electronic active hearing protectors are designed to amplify quiet sounds, attenuate loud sounds, and protect the subject from excess noise exposure. The signal levels that activate the amplification or the attenuation circuitry depend on the design parameters selected by the manufacture, and its intended use. The ANC ear muff amplified quiet signals, and the highest amplitude sound recorded with this muff was 87 $\mathrm{dB}$ measured for the $1 / 3^{\text {rd }}$ octave band centered at $80 \mathrm{~Hz}$ at a velocity of $20 \mathrm{~km} / \mathrm{h}$ (Fig. 6). As shown in Fig. (3) the corresponding signal level was $78 \mathrm{~dB}$ without the ANC muff, thus in this instance the signal was amplified about 9 dB. Thus, at low velocities the ANC circuitry would heighten the audibility of vehicular traffic noise and highway warning sounds. At velocities of $40 \mathrm{~km} / \mathrm{h}$ and above the ANC ear muff attenuation circuitry was engaged, and peak noise levels were limited to $82 \mathrm{~dB}$ or less. At $120 \mathrm{~km} / \mathrm{h}$ the ANC muff reduced the peak noise levels measured by $26 \mathrm{~dB}$. However, the perceived attenuation was much greater than this level because of the much lower noise levels measured in the range from $200 \mathrm{~Hz}$ to $10,000 \mathrm{~Hz}$. That is, in the frequency range where human hear is most sensitive, the ANC muff strongly reduced the measured noise levels. For example at $1 \mathrm{kHz}$, the observed signal level with the ANC ear muff at $120 \mathrm{~km} / \mathrm{h}$ was $35 \mathrm{~dB}$ was less than measured with the half-helmet alone. Fig. (7) displays a waveform created 


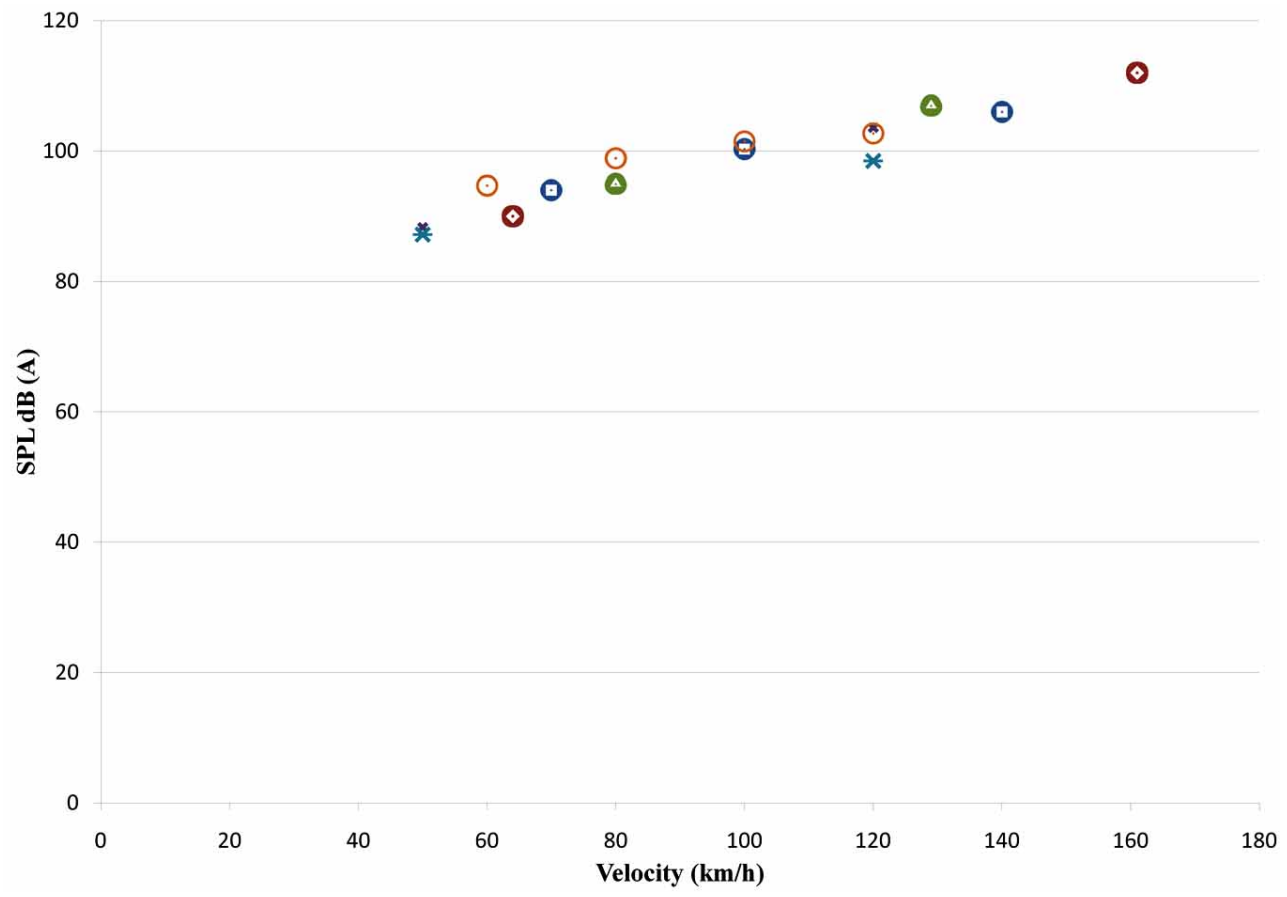

Fig. (5). Helmet noise as a function of velocity for five studies \{purple and blue X for full-face and open face helmets respectively [6], green triangles [25], red squares [26], blue diamond [34], the present data is shown with tan circles\}.

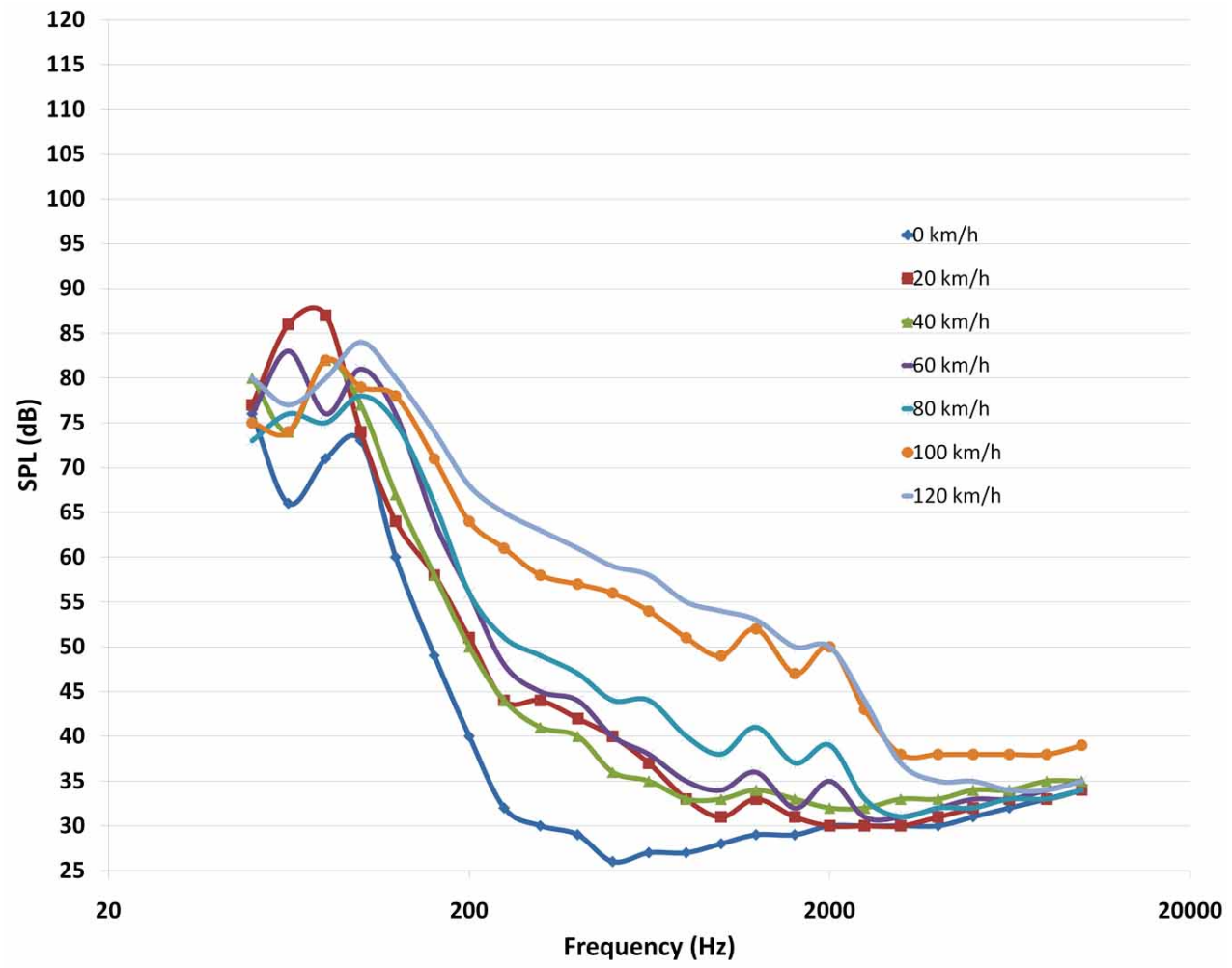

Fig. (6). Sound pressure levels as a function of velocity for a half helmet with the ANC ear muff.

by splicing together 2 second noise samples recorded at each of the seven target velocities from $0 \mathrm{~km} / \mathrm{h}$ to $120 \mathrm{~km} / \mathrm{h}$. The reader can click on the speaker icon and hear the noise levels recorded by the Neumann KU-100 at all velocities from 0 $\mathrm{km} / \mathrm{h}$ to $120 \mathrm{~km} / \mathrm{h}$. These recordings were conducted with settings identical to those used in Fig. (4). [Note to reader. Because the signal level was low with the ANC muff relative to the values shown in Fig. (4), the gain on the reader's sound card may have to incremented to be able to hear these sound samples. Furthermore, if the reader's computer speaker has a weak low-frequency response, $(200 \mathrm{~Hz}$ and below) the reader may be unable to hear this sound file without the use of earphones]. 


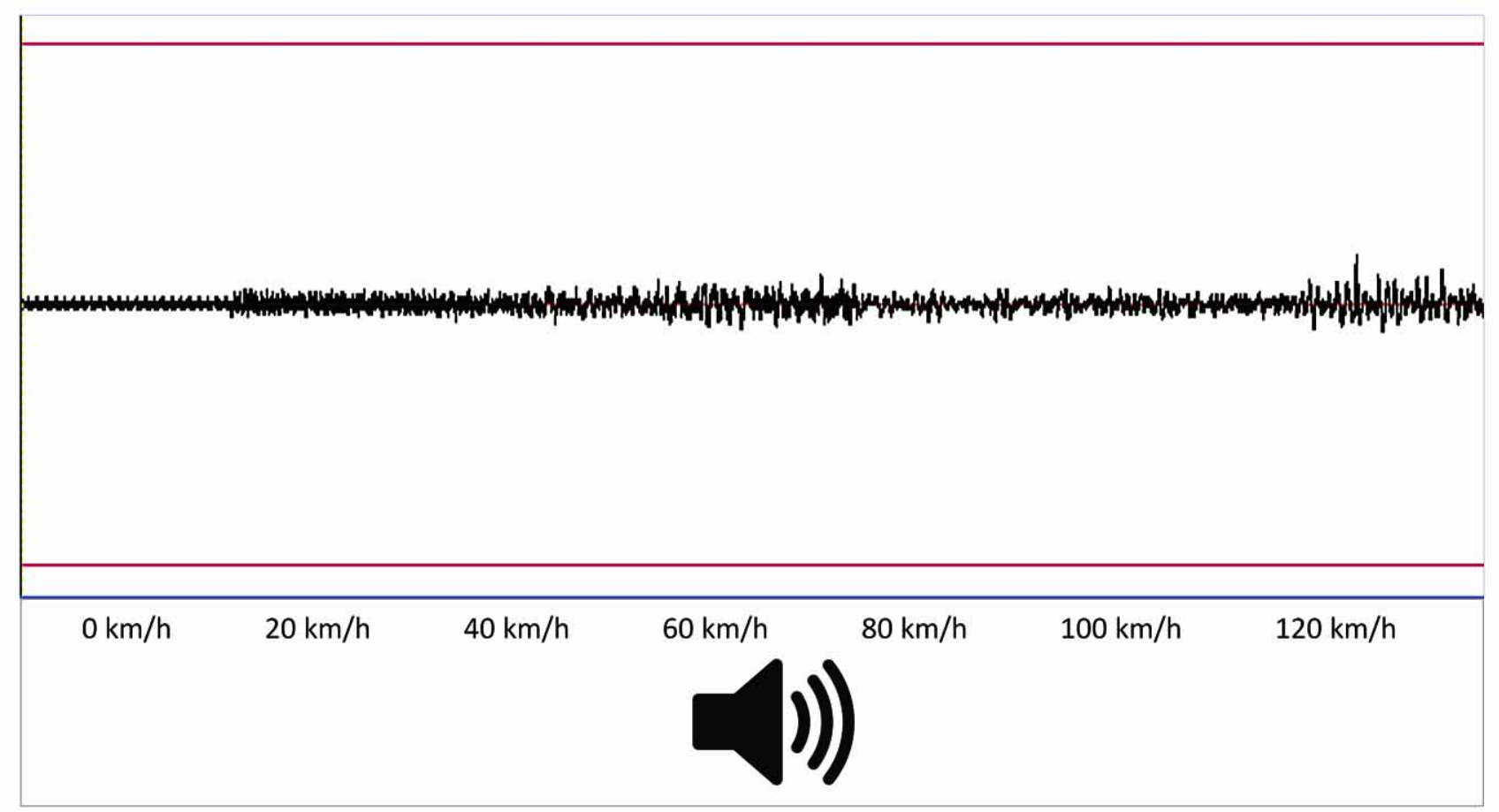

Fig. (7). Waveform created by splicing two-second noise sample for all seven velocities from $0 \mathrm{~km} / \mathrm{h}$ to $120 \mathrm{~km} / \mathrm{h}$ for samples recorded with the active noise control ear muff.

Lower et al. $[32,33]$ also experimented with active noise control earmuffs for motorcycle helmet noise reduction. In wind tunnel tests they found that an aviation pilot's helmet was able to restrict noise levels to $80 \mathrm{~dB}$ (A) at $115 \mathrm{~km} / \mathrm{h}$. However, when they attached an active ear muff to a motorcycle helmet very little active noise reduction was achieved. They proposed that the problem was due to the transmission of vibrations from the structure of the helmet shell and liner to the active muff. The ANC muff used in the present study was also able to attenuate wind noise (Fig. 8). The present results, like those of Lower and his colleagues $[32,33]$ showed that wind noise could be well controlled by active noise control technology, and the observed sound levels were measured at $80 \mathrm{~dB}(\mathrm{~A})$ or less for all velocities tested. Fortunately, ANC technological development has been significant, and many products available today were unavailable to Lower et al. [32, 33]. The ANC muff used in the present study was successfully adapted to the motorcycle helmet; it worked in a real world setting on a moving motorcycle where vibration from the road, and the motorcycle engine were certainly present. We observed no issues that would suggest that the design of the current muff could not be successfully integrated into many styles of motorcycle helmets of current manufacture. Subjectively the quality of the sound transmitted through the muff was a peculiar low-frequency howl. It sounded strange, but it was much quieter than the condition without ANC. We are not certain that the $\mathrm{dB}$ (A) scale is optimal for helmet noise assessment. In the present study the frequency peak of the noise observed with the active muff was well below $160 \mathrm{~Hz}$. The $\mathrm{dB}$ (A) scale is not flat, and this scale diminishes the contribution of both low- and high-frequency sounds to the resulting noise measurement. Moderate noise levels confined to very low-frequencies (such as those in Fig. 6) will be measured as low amplitude signals with the $\mathrm{dB}$ (A) scale. In the present situation, it seemed to us, that the $\mathrm{dB}(\mathrm{A})$ scale underestimated our perception of the loudness of the lowfrequency noise we heard through the muff. This perception, however, is clearly a subjective matter, and different observers with different ears may judge the perceived level of loudness differently.

The ANC muff used in the present study was produced by Pro Tech Technologies, Inc for attachment to a hard hat in noisy industrial settings, and it was not developed specifically for the motorcycle helmet industry. We believe that this type of technology is readily adaptable to the problem of motorcycle helmet noise abatement, and motorcycle helmets with integrated ANC ear muffs may be designed to address both hearing conservation and highway safety objectives. ANC technology can amplify highway warning sounds at the relatively low noise levels associated with low velocities at which most motorcycle collisions occur [37-41], and attenuate wind noise generated at higher velocities. Because the muff or helmet speaker is part of the noise control technology, these helmets can also safely accommodate the need for in-helmet communication. ANC technology permits an enormous range of parameters that can be explored to tackle the helmet noise problem. A noise cancelling full-coverage helmet was first patented in 1997 [42], and hybrid feedforward/feedback noise reduction designs are under development [43]. ANC technology can successfully attenuate wind noise, and it also provides an acoustic link for an improved presentation of highway safety sounds, and communication signals. 


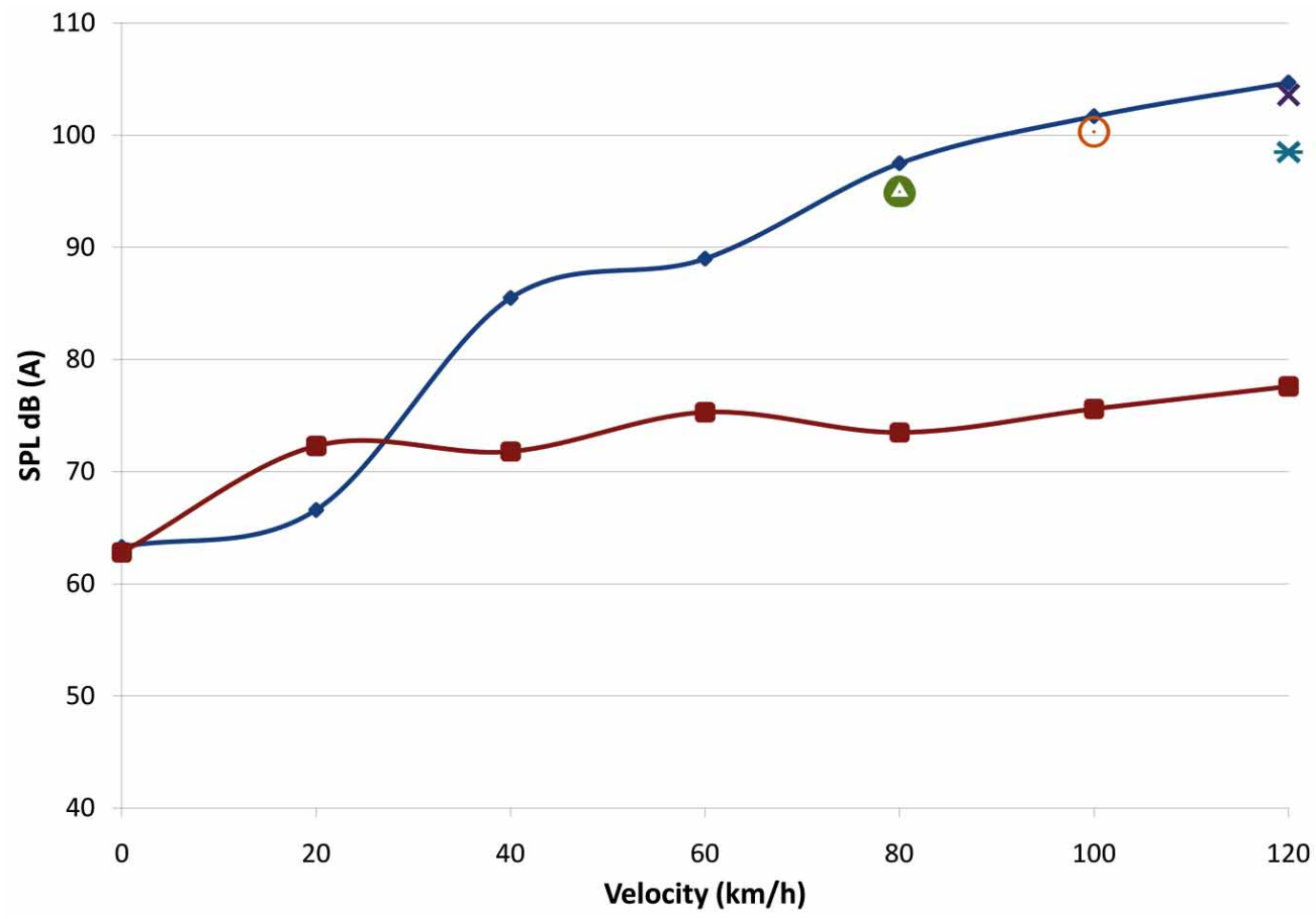

Fig. (8). Helmet noise as function of velocity. Measurement for the half-helmet as shown in blue diamonds, and measurements for the active noise control muff is shown in red squares. For purposes of comparison data at comparable velocities are shown for McCombe et al. [25] green triangle, Alderman et al. [29] orange circle, and Jordon et al. [6] purple X and blue X for full-face and open-faced helmets respectively.

\section{EXPERIMENT 2: SUBJECTIVE PERCEPTIONS OF HELMET NOISE AND THE USE OF EAR PLUGS BY MOTORCYCLISTS}

To our knowledge no research has attempted to survey the perceptions of helmet noise motorcyclist's experience, nor their use of earplugs. Thus all the information in the literature on the noise perceptions of cyclists and earplug use is anecdotal.

\section{Materials and Methods}

A brief 12-item questionnaire was developed and posted on-line using surveymonkey polling software. Participants were solicited using email listserves and several discussion boards catering to motorcyclists (e.g., Cycle Forums, Motorcycle Forum, Motorcycle USA). The call for the survey invited motorcyclists to voluntarily participate in a research project investigating motorcycle riding and the use of helmets. Participation was strictly voluntary and participants received no compensation for their involvement. Responses were collected from October 2009 until May 2010.

\section{RESULTS AND DISCUSSION}

During the sample interval 126 cyclists responded to the survey. Respondent demographics were as follows: The majority of the respondents were male (118 male, and 8 female). The respondents tended to be seasoned riders: $45.2 \%$ had ridden for 10 or more years. A significant proportion of the respondents rode their motorcycle on a daily basis $(42.9 \%)$, and $49.2 \%$ rode their motorcycle a 100 or more miles a day at least once a month. In the present sample $84.9 \%$ use full-face or modular style helmets, and only $0.8 \%$ did not wear helmets.

The main findings are shown in Fig. (9). Panel A: the noise levels associated with motorcycling was perceived as being too loud $(92.1 \%$ report that the noise levels encountered while cycling is unpleasant or disturbing at least occasionally, and $23 \%$ report that it is unpleasant much or most of the time). Panel B: the majority of the riders surveyed use ear plugs or ear bud speakers when motorcycling $(63.5 \%)$. Panel $\mathrm{C}$ : nearly half of the riders surveyed $(46.8 \%)$ reported the experience of a tinnitus (a ringing sensation in their ears). Panel D: the majority of motorcyclists indicated that they were interested in owning a motorcycle helmet that dramatically reduced noise (95.2\%).

\section{Comment Section}

The survey included a comment section and $24.6 \%$ of the respondents submitted comments. Many of their remarks addressed their experience with motorcycle noise levels, a few of their remarks are provided below.

- $\quad$ "A noise cancelling helmet would be a great feature, to only cancel out the low frequency/high amplitude range (roar and rumble) but still allow hearing cars, horns, sirens. Etc."

- $\quad$ "My tinnitus gets much worse after riding if I don't wear earplugs, however, when wearing earplugs I don't suffer from it." 


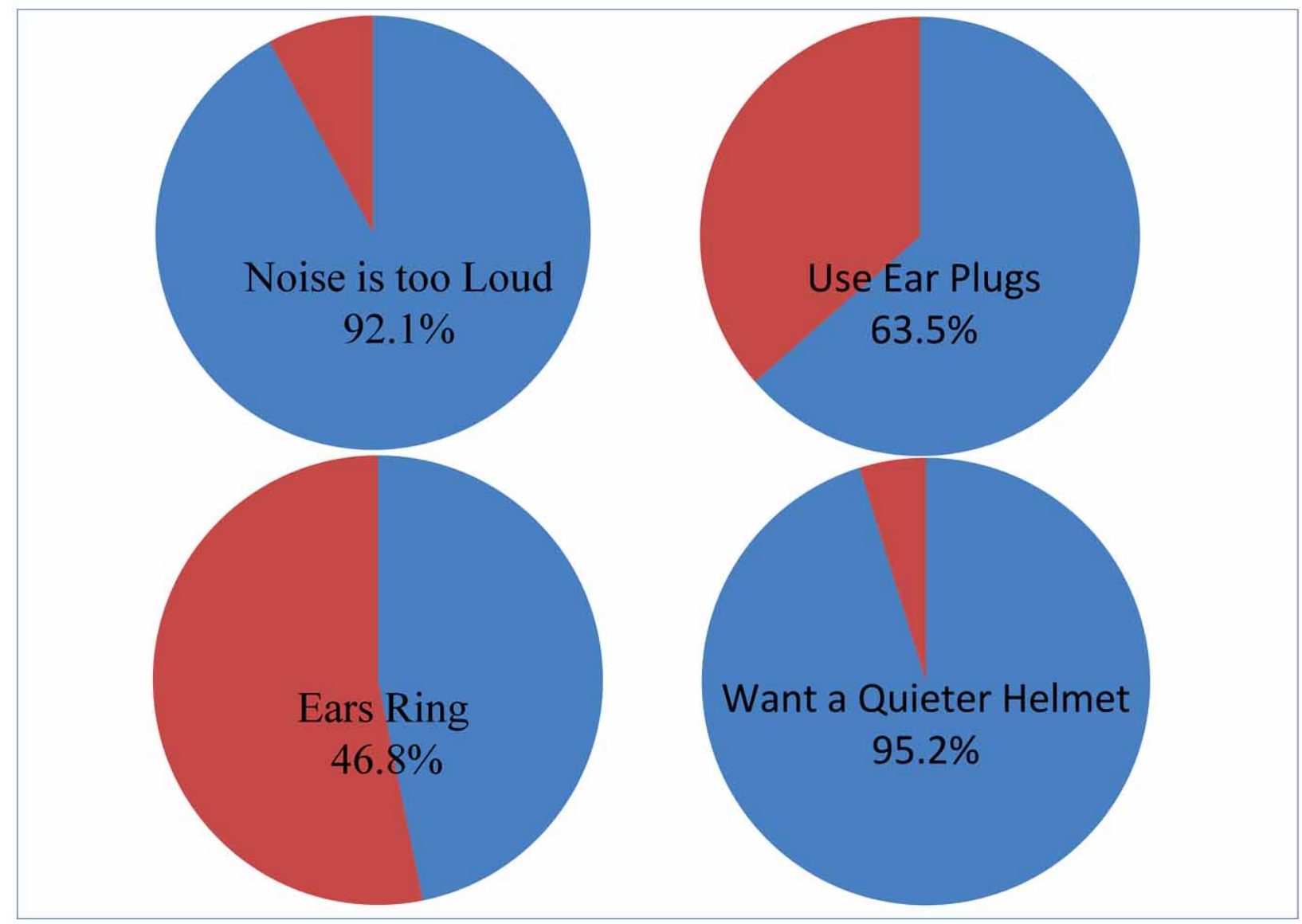

Fig. (9). The results of the on-line survey show that noise levels are perceived as too loud for most riders at least part of the time (panel A), over half of the sample used ear plugs part of the time (panel B), nearly half of the sample experienced ringing in the ears (panel C), and most would be interested in the development of significantly quieter helmets (panel D).

- $\quad$ "Wind noise is a frequent complaint from the riders I've talked to. My helmet tends to be quieter than most, so it's not one of my main concerns."

"I have three helmets, and with my sportbike only one of them is very noisy, and with that helmet I ALWAYS wear earplugs. With the others I wear earplugs at the track and on any longer trip, but the Arai is quiet enough on the CBR to not need it for trips less than an hour if speeds $<75 \mathrm{mph}$."

- "I developed tinnitus from the turbulence off my windscreen. Even earplugs didn't help, as the noise went right into my skull. Between a carbon Kevlar helmet, $30 \mathrm{~dB}$ custom made earplugs, and a newer windscreen, I don't have any further degradation in my hearing."

- "Wind noise is one of my most pressing concerns about the health effects I face from riding a motorcycle. I would be interested in any technology aimed at reducing the problem."

\section{GENERAL DISCUSSION AND CONCLUSIONS}

The laws governing the use of in helmet noise attenuation, communication and entertainment technology (earplugs, ear buds or in-ear speakers, and helmet speakers) vary widely by jurisdiction. In the UK and most of Europe hearing conservation for motorcyclists is strongly supported. Jordan and his associates [6] observed that occupational motorcyclists including police officers, couriers, paramedics and journalists are exposed to daily noise exposure levels of $90 \mathrm{~dB}$ (A) to $103 \mathrm{~dB}$ (A). European standards for noise in the work environment restrict permissible noise levels to $87 \mathrm{~dB}$ (A) or less [44], and motorcyclists are encouraged to wear earplugs [5] until helmets with active noise reduction technology become widely available [43]. There is good reason to be concerned about the risk of noise induced hearing impairment caused by helmet noise. A one hour ride at $80 \mathrm{mph}$ can induce a temporary threshold shift of $11 \mathrm{~dB}$ at $1 \mathrm{kHz}$ [24], and studies suggest that $45 \%$ of Grand Prix motorcyclists, $36 \%$ of motorcycle paramedics, and $6 \%$ of motorcycle driving instructors suffer noise induced hearing loss $[3,5,6]$. Noisy helmets clearly pose a risk for hearing damage, and they are also expected to slow reaction times, impede attention, and reduce the effectiveness of perceptual and behavioral responses pertinent to highway safety [8-11]. Within the United States the laws governing the use of in helmet noise attenuation, communication and entertainment technology vary between states or even municipalities [45]. It is illegal to use or have in your possession helmet speakers in Massachusetts or Rhode Island; helmet speakers are legal for communication purposes only in Georgia, Illinois, and Pennsylvania. Helmet speakers in one ear are legal in 
California, Maryland, Minnesota, and New York. Maryland prohibits earplugs in both ears unless they are custom made. The California earplug law (originally like the Maryland ordinance) was changed in 2004 to permit use of most earplugs including disposable earplugs. Most jurisdictions prohibit binaural ear buds. In Oregon the earplug, ear bud, and helmet speaker laws vary by municipality. In Pennsylvania it is illegal to use a passive or electronic device intended to impair hearing (reduce noise). Overall, the laws in the US appear to embrace the idea that hearing in helmets should be undisturbed to promote traffic safety. Outside of the endorsement of earplug use in California there is little attention directed towards hearing conservation in US motorcycle laws, though many motorcycle journalists strongly encourage the use of earplugs. The initial steps towards the development of a motorcycle helmet acoustic testing protocol have been developed for the European community [28], but this effort has focused principally on hearing conservation rather than on improving the detection of highway warning sounds. The interests of hearing conservation and highway safety would be well served by the development of an acoustic testing protocol for employment within the United States. Optimally, this protocol should address helmet noise control, the perception of highway warning signals, and the perception of vehicular noise that may alert the cyclists to changes in the flow of the surrounding traffic. The Neumann KU-100 is suitable for both helmet noise measurements and for the development of binaural sound files to be used for testing the ability of listeners to perceive highway warning sounds and the noise produced by vehicular motion. In our laboratory we have used the Neumann dummy head to measure hearing in football helmets [46], and the Neumann dummy head has also been used to study the ability of blind pedestrians to detect the motion of hybrid vehicles [47]. The adoption of an objective acoustic testing protocol for motorcycle helmets would encourage the active noise control and safety helmet industries to cooperatively develop new products that would address the helmet noise problem. The creation of a voluntary standard for acoustic certification for motorcycle helmets (much like the Snell safety certification for motorcycle helmets) would serve the interests of the Department of Transportation, the helmet industry, hearing conservation interests, and other stake holders invested in the promotion of health and highway safety. An acoustic certification standard for motorcycle helmets would likely resolve the discrepancy in the US laws regarding in-helmet noise control technology and communication.

At present no active noise control motorcycle helmets are current available. Because one source of helmet noise is produced by the intrusion of turbulent air around the neck of a rider wearing a full-face helmet, some helmets employ a passive noise barrier inserted around the chin and neck, and this approach can reduce helmet noise by approximately 6 $\mathrm{dB}[32,33]$. However, airflow in and out of the helmet is also a critical consideration. The reduction of air flow within a helmet can lead to a buildup of $\mathrm{CO}_{2}$ [48], and heat [49], and these factors could lead to impaired cognitive performance. Full-face motorcycle helmets and the measurement of cognitive performance is receiving increased attention [50], and helmets with good airflow and active noise control technology appear to be positioned to receive a strong demand from the consumer.

With increases in urban congestion, and increments in the cost of gasoline more individuals are likely to adopt motorcycle transportation, and consequently the incidence of motorcycle fatalities is expected to rise more swiftly than the 4 -fold increase reported over the past 15 years [12]. We use our hearing as an early warning system, our hearing can alert us and propel us to scan our surroundings to better assess changes in the flow of traffic. Visual perception is both more accurate and faster when the visual stimulus is paired with an auditory event [16-18]. Because motorcycle helmets are noisy at even moderate velocities, the acoustic capacity of motorcyclists is limited, and the use of earplugs to attenuate helmet noise can impair the motorcyclist's acoustic awareness even further. The incidence of collisions between motorcyclist and other vehicles may be reduced by improving the ability of cyclists to hear highway warning sounds, and respond to unexpected incursions from the surrounding traffic. Motorcycle helmets with ANC technology show strong potential to address hearing conservation and highway safety objectives.

\section{SUPPORTIVE/SUPPLEMENTARY MATERIAL}

Supplementary material is available on the publishers Web site along with the published article.

\section{REFERENCES}

[1] Johngepier GAM, Van der Weerd A. Research into the noise hazards and loss of hearing in motorcycle patrols of the state police. The Hague: Department of Research and Development 1989.

[2] Ross BC. Noise exposure of motorcyclists. Ann Occup Hyg 1989; 33: $123-7$.

[3] McCombe AW, Binnington J. Hearing loss in Grand Prix motorcyclists: occupational hazard, or sports injury?. Br J Sp Med 1994; 28: 35-7.

[4] McCombe AW, Binnington J, Davis A, Spencer H. Hearing loss and motorcyclists. J Laryngol Otol 1995; 109: 599-604.

[5] McCombe AW. Hearing loss in motorcyclists: occupational and medicolegal aspects. J Royal Soc Med 2003; 96: 7-9.

[6] Jordan C, Hetherington O, Woodside A, Harvey H. (2004). Noise induced hearing loss in occupational motorcyclists. J Environ Health Res 2004; 3: 373-84.

[7] Lesage F-X, Jovenin N, Deschamps F, Vincent S. Noise-induced hearing loss in French police officers. Occup Med (Oxf) 2009; 59: 483-6.

[8] Gilden DL, Thornton T, Mallon MW. 1/f noise in human cognition. Sci 1995; 267: 1837-9.

[9] Hygge S. The interaction of noise and mild heat on cognitive performance and serial reaction time. Environ Intern 1991; 17: 22934.

[10] Saha S, Gandhi A, Das S, Kaur P, Singh SH. Effect of noise stress on some cardiovascular parameters and audiovisual reaction time. Indian J Physiol Pharmacol 1996; 40: 35-40.

[11] Sanford A J. (1971). Effects of changes in intensity of white noise on simultaneity judgments and simple reaction times. Q J Exp Psych A 1971; 23: 296-303.

[12] National Highway Traffic Safety Administration. Traffic Safety facts: Data Motorcycles 2006.

[13] Huang B, Preston J. A literature review on motorcycle collisions: Final report. Transportation Studies Unit: Oxford University 2004.

[14] Preusser DF, Williams AF, Ulmer RG. (1995). Analysis of fatal motorcycle crashes: crash typing. Accid Anal Prev 1995; 27: 84551 
[15] National Highway Traffic Safety Administration, Traffic Safety facts: Data Motorcycles 2008.

[16] Knudsen E I, Knudsen PF. Vision guides the adjustment of auditory localization in young barn owls. Sci 1985; 230: 545-8.

[17] Kubovy M, Valkenburg DV. Auditory and visual objects. Cogn 2001; 80: 97-126.

[18] Perrott DR, Saberi K, Brown K, Strybel T Z. Auditory psychomotor coordination and visual search performance. Percept Psychophy 1990; 48: 214-26.

[19] Harrison RT. The effectiveness of motorcycle helmets as hearing protectors. J Acoust Soc Am 1973; 53: 366.

[20] Henderson R. Effect of safety helmets on auditory capability. Report No. 801759, National Highway Traffic Safety Administration: Washington DC 1975.

[21] Purswell JL, Dorris AL. Warnings and human behavior: Implications for the design of product warnings. J Prod Liability 1977; 1: 255-63.

[22] Van Moorhem WK, Shepherd KP, Magleby TD, Torian GE. Acoustics of motorcycle helmets. J Acoust Soc Am 1977; 6: S25.

[23] McKnight AJ, McKnight AS. The effects of motorcycle helmets upon seeing and hearing. Accid Anal Prev 1995; 27: 493-501.

[24] McCombe AW, Binnington J, McCombe TS. Hearing protection for motorcyclists. Clin Otolaryngol and Allied Sci 1993; 18: 465-9.

[25] McCombe AW, Binnington J, Nash D. Two solutions to the problem of noise exposure for motorcyclists. Occup Med (Oxf) 1994; 44: 239-42.

[26] Binnington JD, McCombe AW, Harris M. Warning signal detection and the acoustic environment of the motorcyclist. Br J Audiol 1993; 27: 415-22.

[27] Hancock PA, Wulf G, Thom D, Fassnacht P. Driving workload during difficult driving maneuvers. Accid Anal Prev 1990; 22: 28190.

[28] Gillingham S, Cesari D, Otte D, Chinn B. COST 327, Motorcycle Safety Helmets, Section 8.6 Motorcycle Aerodynamic Noise, pp. 219-24, 1999.

[29] Van der Weerd A. Comparative sound measurements with alternative motorcycles, fairings and helmets on behalf of the state police. The Hague: Department of Research and Development 1990.

[30] Van der Weerd A. Comparative research into the noise hazard to motorcyclist with various fairings, shields and helmets. The Hague: Department of Research and Development 1990.

[31] Lower M, Hurst DW, Claughton A, Thomas A. Sources and levels of noise under motorcycle helmets. Proc Inst Acoust 1994; 16: 31926.

[32] Lower M, Hurst DW, Thomas A. Noise levels and noise reduction under motorcycle helmets. Proceedings of Internoise '96, Book 2, pp. 979-82.

[33] May JJ. Occupational hearing loss. Am J Med 2000; 37: 112-20.
[34] Aldman B, Gustaffson H, Nygren A, Wersall J. Hearing and motorcycle helmets. J Traffic Med 1983; 11: 42-4.

[35] Mlyński R, Kozlowski E, Žera J. Attenuation of noise by motorcycle safety helmets. Int J of Occup Saf Ergon 2009; 15: 28793.

[36] Carley M, Walker I, Holt N. (2010). On-road measurement of motorcycle helmet noise mechanisms. In: Bogerd, Carley, Crundall, Otto, Shahar, Shinar, Webb, \& Brühwiler (Eds.) COST Action 357-Accident Prevention Options with Motorcycle Helmets Final Report. Empa, St. Gallen, Switzerland 2010; p. 45.

[37] Hurt HH, Ouellet JV, Thom ER. Motorcycle accident cause factors and identification of countermeasures, Vol. I. Technical Report DOT HS-5-01160. National Highway Traffic Safety Administration: Washington, DC. 1981.

[38] Francescutti LH, Saunders LD, Hamilton SM. Why are there so many injuries? Why aren't we stopping them?. CMAJ (Ottawa) 1991; 144: 57-61.

[39] Road Safety Committee. Social development committee inquiry into motorcycle safety in Victoria: Victoria, Australia 1992.

[40] Road Safety Committee. Inquiry into motorcycle safety in Victoria: Victoria, Australia 1993.

[41] Road Safety Committee. Inquiry into the review of motorcycle safety in Victoria: Victoria, Australia 1998.

[42] Lazzeroni JJ, Carevich MK. Noise cancelling microphone for full coverage style helmets. U.S. patent 5684 880, November 4, 1997.

[43] Castañé-Selga R, Peña RS. Active noise hybrid time-varying control for motorcycle helmets. IEEE Trans Control Sys Technol 2010; 18: 602-12.

[44] Official Journal of the European Community, "Directive 2003/10/EC, noise at work regulations," UE-2003/10/EC, 2003.

[45] American Motorcycle Association: State Motorcycle Laws.

[46] Gordon MS, Brown CH, Kolbusz M. Hearing within rigid constraints: Speech detection while wearing a football helmet. The Open Acoust J 2010; 3: 1-10.

[47] Garay-Vega L, Hastings A, Pollard JK, Zuschlag M, Sterns MD. Quieter cars and the Safety of blind pedestrians: Phase I. DOT HS 811 304, April, 2010.

[48] Brühwiler PA, Stämpfli R, Huber R, Camenzind M. CO2 and O2 concentrations in integral motorcycle helmets. Appl Ergon 2005; 36: 625-33.

[49] Bogerd CP, Brühwiler PA. Heat loss variations of full-face motorcycle helmets. Appl Ergon 2009; 40: 161-4.

[50] Bogerd CP, Strässle K, Brühwiler PA. Laboratory subject study of cognitive effects when wearing a full-face motorcycle helmet in warm conditions. In: Bogerd, Carley, Crundall, Otto, Shahar, Shinar, Webb, \& Brühwiler (Eds.) COST Action 357-Accident Prevention Options with Motorcycle Helmets Final Report. Empa, St. Gallen, Switzerland 2010; pp. 48-9. 